\title{
Effect of Nitriding on Wear Behavior of Graphite Reinforced Aluminum Alloy Composites
}

\author{
Bhujang Mutt Girish, Bhujang Mutt Satish, Hanyalu Ramegowda Vitala
}

Research and Development Center, Department of Mechanical Engineering, East Point College of Engineering and Technology, Bangalore, Karnataka, India.

Email: bmgiri1@gmail.com

Received April 22 $2^{\text {nd }}, 2011$; revised May 20 ${ }^{\text {th }}, 2011$; accepted May 29 ${ }^{\text {th }}, 2011$.

\begin{abstract}
The paper evaluates the effect of nitriding on the wear behavior of graphite reinforced aluminum 6061 alloy composites. The composites were prepared using the liquid metallurgy technique. The content of graphite in the composites was varied from 3 to $7 \%$ (by weight) in steps of $2 \%$. The nitriding process was carried out at $500{ }^{\circ} \mathrm{C}$ for 24 hours. Three categories of specimens, namely, the nitrided composites, non-nitrided composites, as well as the alloy specimens were tested for their wear behavior. Pin-on disc equipment was used for wear testing. X-Ray Diffraction technique (XRD) was used to confirm the implantation of nitrogen in the composites. It was observed that the nitrided composites have better wear resistance than the non-nitrided composites.
\end{abstract}

Keywords: Composites, Nitriding, Surface Engineering, Wear

\section{Introduction}

Aluminum (Al) and its alloys posses numerous advantages such as low specific weight, high strength-toweight ratio and low cost, and hence find wide applications in the automotive, aviation and space industries [1]. However, the main limitation of their wider application for commercial purpose is the lack of surface hardness, mechanical strength and wears resistance, together with low thermal and chemical stability. Therefore, a surface modification with the aim to improve the above properties is necessary for further industrial use. This can be achieved by formation of aluminum nitrite (AlN) on $\mathrm{Al}$ surface, obtained by nitrogen insertion into the surface of bulk $\mathrm{Al}$ as AIN exhibits excellent tribological properties combined with high thermal and chemical stability [2].

The use of aluminum for light weight machine parts and car components has recently increased, although non-metallic materials, such as resin, are also used for this purpose. Al alloys have advantages over non-metallic materials such as light weight, corrosion-resistance, and are workable and have good thermal conductivity. However, the hardness, wear and seize resistance of $\mathrm{Al}$ alloys is lower than that of the steel, and hence there is a limit on the application as sliding parts. Thus, research has been carried out on surface modification technology to increase the applicability of $\mathrm{Al}$ alloys as sliding parts [3].

MMCs (Metal Matrix Composites) have received increasing attention in the recent decades as engineering materials. MMCs are primary candidate materials for industrial applications in the aerospace, automotive and power utility industries. However, their properties such as strength, toughness, and wear and corrosion properties depend to a great extent on several factors of which matrix properties are very important [4].

A more widespread use of light metal alloys in tribological applications like guide bars, bearing plates, seat supports or bushings demands powerful functional surface coatings to provide wear protection as well as compressive strength. The direct contact of uncoated light metal substrates with sliding or oscillatory counterparts results in severe wear, seizing and high frictional coefficient, even under lubricated conditions.

There are different surface treatment processes available commercially for aluminum alloys to increase the corrosion and wear resistance, such as nitriding, anodic oxidation, electrolysis, nickel plating etc. Nitriding is one surface modification technique that is widely used to increase the fatigue, mechanical strength and wear resistance of machine parts of carbon or alloys steels. It is a process of introducing nitrogen in to the metals so as to 
modify the surface properties, and increase the hardness, wear resistance and corrosion resistance [5].

The present paper aims to report the evaluation of wear behavior of nitrided aluminum MMCs reinforced with graphite particles and the same is compared with non- nitrided MMCs.

\section{Experimental Method}

\subsection{Preparation of Composites}

Aluminum 6061 alloy with the chemical composition given in Table $\mathbf{1}$ was used as the matrix material. The optical emission spectrometer was used determine the chemical composition of the alloy. The reinforcement material used was graphite which is a solid lubricant with adequate resistance to wear. The composites were fabricated by liquid metallurgy technique, the details of which are available elsewhere [6]. Graphite particles (0\%, 3\%, $5 \%$ and $7 \%$ by weight) were heated separately to a nominal temperature to remove moisture content if any, and introduced into the vortex of the effectively degassed $\mathrm{Al}$ 6061 molten alloy. The molten alloy was stirred rigorously using a stirrer having a ceramic coated steel impeller.

\subsection{Nitriding of Specimens}

Nitriding is a promising method for surface treatment to improve hardness, corrosion, wear and fatigue resistance of materials. Nitriding process can improve the tribological and mechanical properties by enriching the near surface region with nitrogen. Nitrides produced by the combination of nitrogen with alloying elements posses a higher hardness as compared with the iron nitrides. It is due to this fact that the nitriding process is primarily used to increase the hardness and wear resistance of the surface of several metals including steel.

The specimens prepared for wear testing were washed in distilled water, and then with acetone and later subjected to the nitriding process. Nitriding was accomplished in a muffle furnace where ammonia gas was introduced into the air-tight furnace chamber heated to a temperature of $500^{\circ} \mathrm{C}$. The specimens were exposed for a duration of 24 hours and the ammonia feed rate was 20CFH (Cubic feed/hr).

Ammonia gas decomposes giving the nitrogen in nascent form or monatomic nitrogen, which is the only form capable of entering the material and diffusing in it.

\subsection{Wear Test Equipment}

The wear tests were conducted in accordance with ASTM G99 standards using a pin-on-disc sliding wear testing machine, which is similar to the one, used by Poonawala et al., [7]. EN24 steel disc of diameter 200 $\mathrm{mm}$ and chemical composition: C-0.45, Si-0.35, Mn-0.70, Cr-1.40, Mo-0.35, Ni-1.80, S-0.05, P-0.05 in weight $\%$, was used as the counter face on which the test specimens slide. Hardness of the steel disc was HRc 57 achieved by oil quenching at $850^{\circ} \mathrm{C}$ and tempering at $550^{\circ} \mathrm{C}$ for 2 hours. Arrangements were made to hold a specimen and also for application of the load on the specimen. The test specimen was clamped in a vertical sample holder and held against the rotating steel disc. In the present investigation, loads of $20-160 \mathrm{~N}$ in steps of $20 \mathrm{~N}$ were used. The rotational speeds employed were 200, 250, and $300 \mathrm{rpm}$, which at an average distance of 80 $\mathrm{mm}$ from the center gave corresponding linear speeds of $1.25,1.56$, and $1.87 \mathrm{~m} / \mathrm{s}$ respectively.

\subsection{Testing of Specimens}

The 'weight loss' method was adopted in the present study in which the pins of the material under investigation were $6 \mathrm{~mm}$ in diameter and $15 \mathrm{~mm}$ in length. A fresh disc was used each time and before each test, the disc was cleaned with acetone to remove any possible traces of grease and other surface contaminants. The specimens were cleaned with ethanol and weighed before and after the tests using a balance accurate to $\pm 0.001 \mathrm{~g}$. The wear results were computed from weight loss measurements. The duration of each test was exactly 60 minutes. The wear volume was calculated from the ratio of weight loss to density and wear rate was calculated using sliding distance and wear volume. Usage of such relations to calculate the wear parameters is common and has been used by Rohatgi et al., [8]. The data for the wear tests was taken from the average of three measurements. The standard deviation was about 5\%. The surfaces of the worn specimens were cleaned thoroughly to remove the loose wear debris and then observed using a scanning electron microscope (SEM). Along with the test specimens, even the surface of the counterpace steel disc was subjected to the SEM analysis in order to draw as much vital information as possible about the wear behavior of the composite specimens.

Table 1. Composition of Al-6061 alloy.

\begin{tabular}{cccccccccccccc}
\hline Elements & $\mathrm{Cu}$ & $\mathrm{Mg}$ & $\mathrm{Si}$ & $\mathrm{Fe}$ & $\mathrm{Mn}$ & $\mathrm{Ni}$ & $\mathrm{Zn}$ & $\mathrm{Pb}$ & $\mathrm{Sn}$ & $\mathrm{Ti}$ & $\mathrm{Cr}$ & $\mathrm{Al}$ \\
\hline \% by Wt & 0.36 & 0.99 & 0.80 & 0.12 & 0.02 & 0.01 & 0.01 & 0.07 & 0.05 & 0.01 & 0.12 & $\mathrm{Bal}$ \\
\hline
\end{tabular}




\section{Results and Discussion}

Wear of composite materials although appears to be simple, the actual process of material removal is quite complex. This can be attributed to the fact that a large number of factors influence wear such as metallurgical factors (weight percentage, chemical composition, and size of the reinforcement) or service (i.e., speed, pressure) or other contacting factors (lubrication, corrosion, etc.) The factors that have been considered in the present study to explain the wear behavior of the composites are the applied load, the applied speed, and the weight percentage of the reinforcement.

Scanning electron microscopy were carried out on samples which were ground to 600 grit $\mathrm{SiC}$ and polished using alumina powders up to $1 \mu$, and etched using Keller's reagent. The microphotograph of the $7 \%$ graphite composite showing uniform distribution of the reinforcement is shown in Figure 1. The Figure 2(a) is the EDX of a nitrided Al6061 alloy specimen. The nitrogen peak is clearly visible indicating the presence of nitrogen. The Figure 2(b) shows the thickness of the coating that was obtained and it was found to be $1.10 \mathrm{~mm}$.

\subsection{Effect of Load and Speed on the Wear Rate}

The specimens tested fall into three categories, namely, the unreinforced and non-nitrided specimen, the nonnitrided composite, and the nitrided composite. Three pin specimens were tested from each category at each specified load and speed.

The results were averaged to obtain the final wear rate, which are presented graphically in Figures 3-5. In the graphs shown in Figure 3-5, 'UN' means unnitrided specimens, while 'nit' means nitrided specimens. The wear rate of the unreinforced alloy is also plotted in order to enable comparison with the other category specimens. It is found from the graphs that the wear rate of the specimens belonging to all the three categories increased with the applied load. The wear rate of the non-nitrided composite as well as the nitrided composite specimen reduced with the increase in graphite content. It is clearly evident from the graphs that there exists a certain load, i.e., a transition phenomena at which there is a sudden increase in the wear rate of the specimens belonging to all the three categories. However, the transition loads for the nitrided composites were much higher than that observed for the non-nitrided composites, and that of the non-nitrided composites was much higher than the unreinforced alloy and also the transition load increased with the increase in graphite particle content.

The Figure 3 presents the behavior of the specimens tested at a speed of $1.25 \mathrm{~m} / \mathrm{s}$ and load ranging from 20 to $200 \mathrm{~N}$. It was observed that the unreinforced matrix alloy

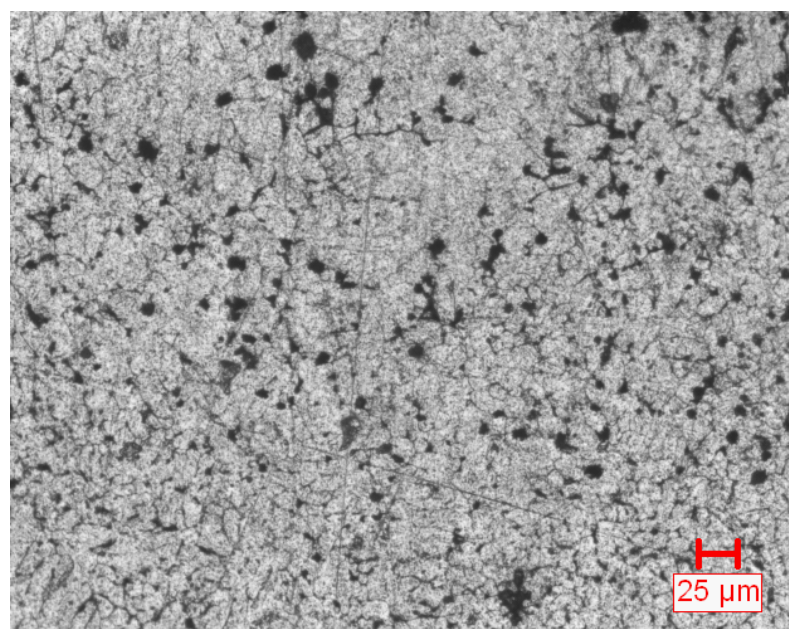

Figure 1. SEM showing uniform distribution of graphite particles in the alloy matrix.

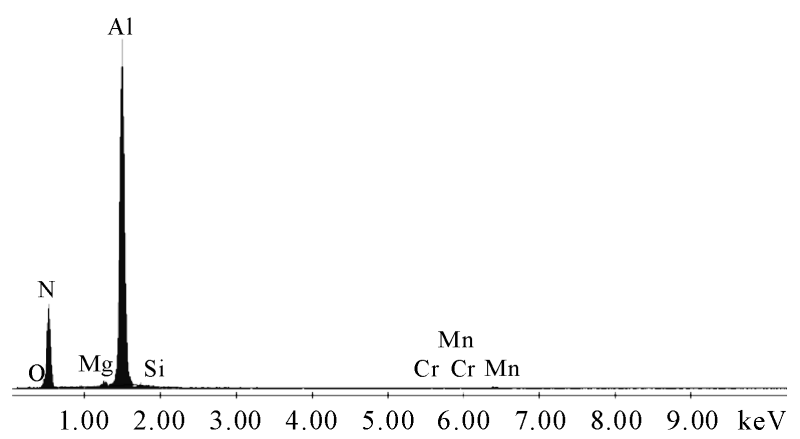

(a)

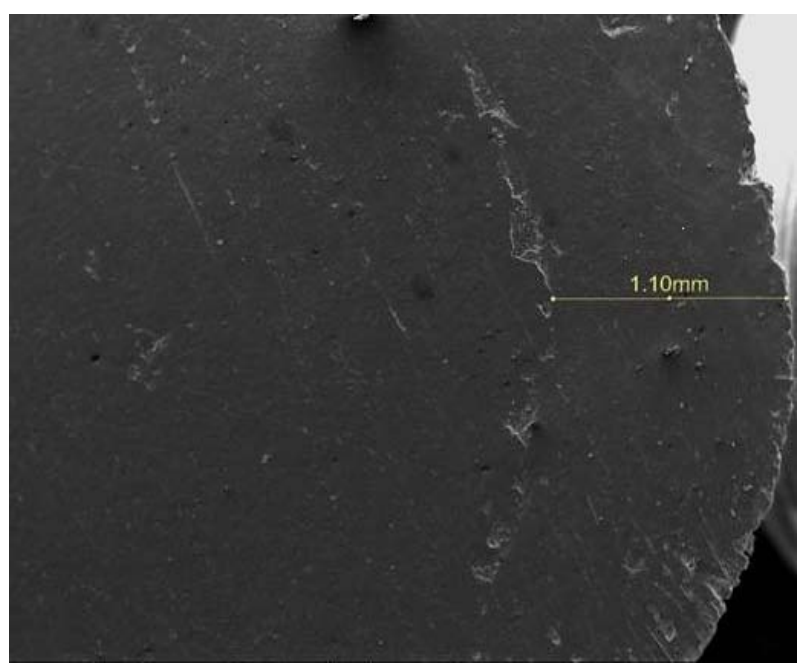

(b)

Figure 2. (a) EDX of a nitrided composite specimen; (b) SEM showing the thickness of the coating on the specimen.

showed a transition from mild to severe wear at a load of $60 \mathrm{~N}$, while the 3 and 5\% graphite reinforced composites showed a transition at 140 and $160 \mathrm{~N}$ respectively. The 


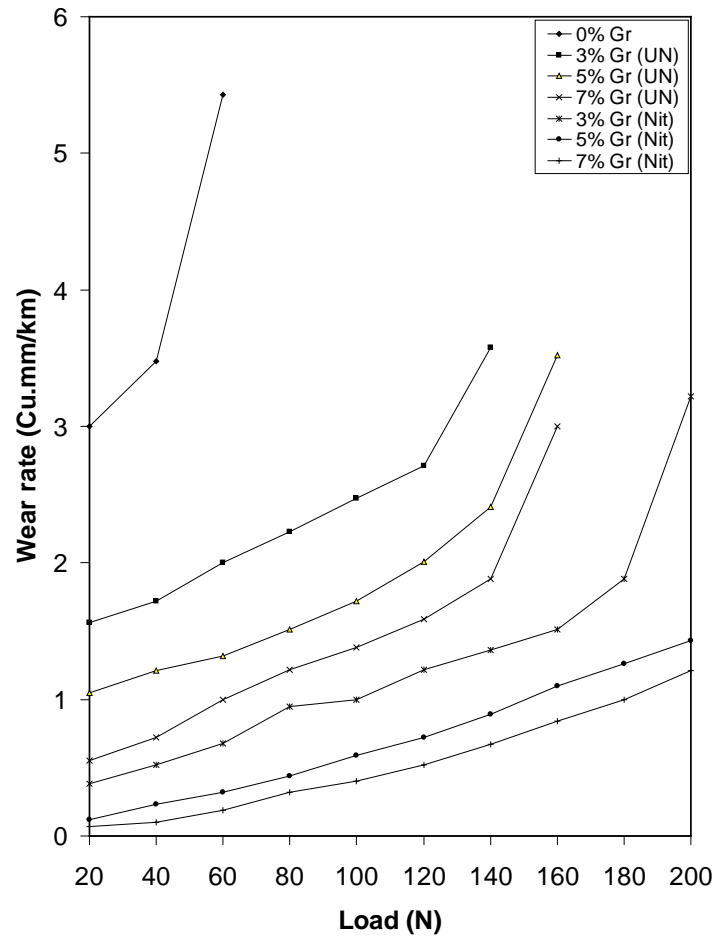

Figure 3. Graph of wear rate v/s load at a speed of $1.25 \mathrm{~m} / \mathrm{s}$.

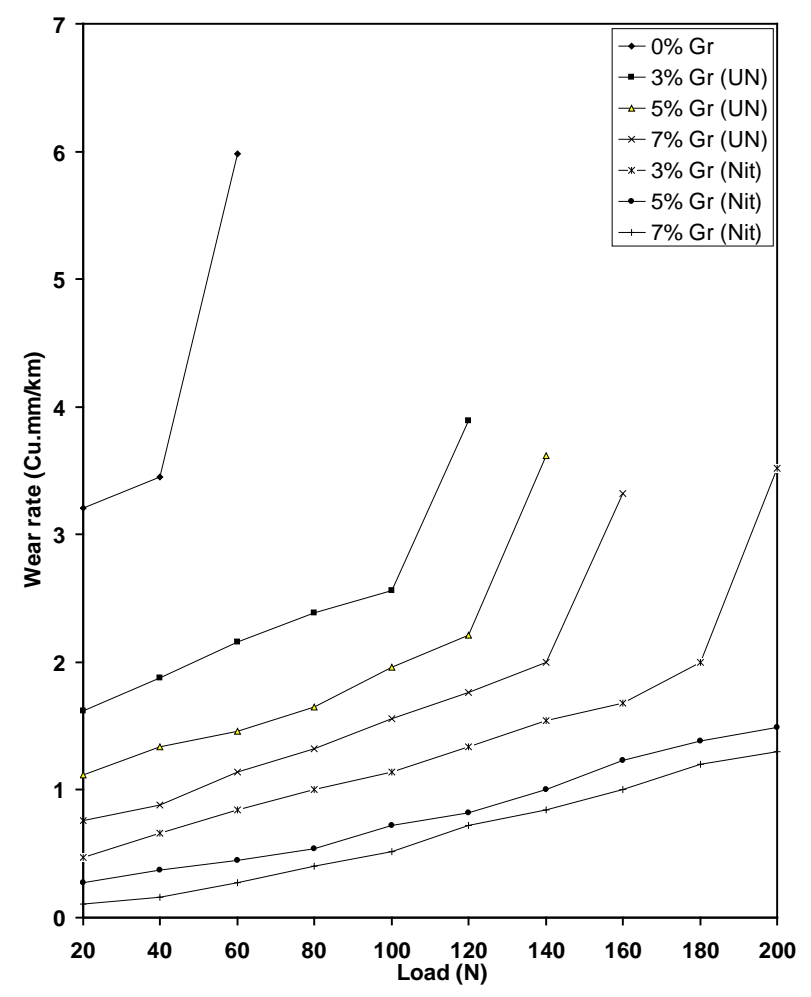

Figure 4. Graph of wear rate v/s load at speed of $1.56 \mathrm{~m} / \mathrm{s}$.

same observation was made at a load of $140 \mathrm{~N}$ in case of $7 \%$ graphite reinforced composites. This observation

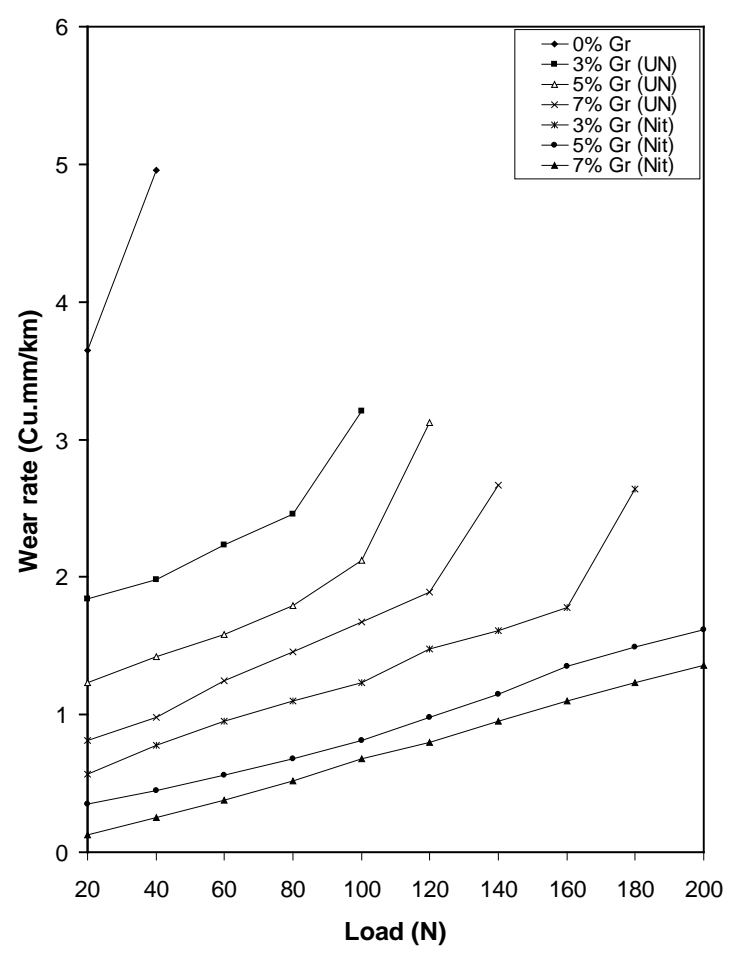

Figure 5. Graph of wear rate v/s load at a speed of $1.87 \mathrm{~m} / \mathrm{s}$.

which is evident from Figure 3 indicates that the presence of graphite reinforcement delays the transition from mild to severe wear, and increases the transition load of the $7 \%$ reinforced composite by almost 2.5 times with respect to the unreinforced alloy. Interestingly, similar transition in the case of nitrided composites was observed at $200 \mathrm{~N}$ in those having a reinforcement content of $3 \%$ graphite, while the 5 and $7 \%$ reinforcement composites show no transition at all even at $200 \mathrm{~N}$.

It follows from the results obtained that comparatively low wear rates exist at lower loads, thereby indicating the regime of mild wear. In this regime of mild wear, the composites demonstrate significant wear resistance than the alloy counterpart. At higher loads, the materials exhibit rapid increase in wear rate. At loads greater than the transition loads, severe wear occurs leading to seizure of the materials. The severe wear manifests itself by a rapid rate of material removal in the form of generation of coarse metallic debris, and also by massive surface deformation and material transfer to the counter face.

The composites behave very differently from the unreinforced alloy. The alloy shows a transition at $60 \mathrm{~N}$ when tested at 1.25 and $1.56 \mathrm{~m} / \mathrm{s}$, while the same is observed at $40 \mathrm{~N}$ in case of $1.87 \mathrm{~m} / \mathrm{s}$ test. Similarly the composite with $3 \%$ reinforcement shows a transition at $140 \mathrm{~N}$ when tested at $1.25 \mathrm{~m} / \mathrm{s}$, at $120 \mathrm{~N}$ when tested at speed of $1.56 \mathrm{~m} / \mathrm{s}$ and $100 \mathrm{~N}$ at $1.86 \mathrm{~m} / \mathrm{s}$. Similar observations for 5 and $7 \%$ composites as well as the nitrided 
composites are clearly evident form the graphs shown in Figures $\mathbf{4}$ and $\mathbf{5}$.

The composite with $5 \%$ graphite shows a transition at $160 \mathrm{~N}$ when tested at $1.25 \mathrm{~m} / \mathrm{s}$, at $140 \mathrm{~N}$ at speed of 1.56 $\mathrm{m} / \mathrm{s}$, and the same is observed at $120 \mathrm{~N}$ in case of $1.87 \mathrm{~m} / \mathrm{s}$ test. The above observations clearly indicate that the sliding speeds employed have a significant effect on the wear rate transition on the materials. The transition in wear rate decreases with the increase in speed in all the materials. The results obtained are on par with the one obtained by Lee, et al., [9] who have reported that the wear mechanisms are strongly dependent on the sliding speeds.

The mild wear of the alloy is oxidation dominated wear at low sliding speeds and loads. In the case of composites, due to the existence of graphite particles, the oxide film of the metal is not continuous and tenacious. It is removed by friction forces in the following sliding friction resulting in oxidation assisted mild abrasion wear. Hence it can be considered that the dominating wear mechanism is the removal and reproduction of the oxide film. This kind of wear is maintained until higher loads are employed, under which condition the wear mechanism transforms from mild to severe wear. The morphologies of wear surfaces of 7\% graphite reinforced composites are shown in Figure 6(a)-(c). Figure 6(a) shows the wear tracks for unreinforced alloy, while Figure 6(b) and (c) represent the wear tracks for composite and nitrided composite respectively. The wear tracks in Figure 6(b) and (c) show typical abrasive wear for the composites tested at low loads of 20 and $60 \mathrm{~N}$ respectively. Hence it can be concluded that the dominating wear mechanism is abrasive wear at low loads.

It was observed that at higher loads a transition occurs from mild to severe wear, and the wear rate quickly increases by tremendous rate. Due to the high loads employed, the friction and wear increased obviously. In this condition, the removal and formation of oxide films are faster than that of mild oxidation, thereby resulting in relatively higher wear rates.

\subsection{Effect of Graphite Particles on Wear Rate}

It follows from the observations that the graphite particles play a very strong role in enhancing the wear resistance of the composites. It was found that the transition load increases with the increase in graphite content and also the wear rate of the composites was lower than that of the base alloy without graphite. This is obviously due to the release of graphite particles by the composite specimens on to the mating surface during sliding which provides resistance to wear. The release of graphite onto the sliding interface causes formation of a thin film such that the relative movement of the mating surfaces pro-

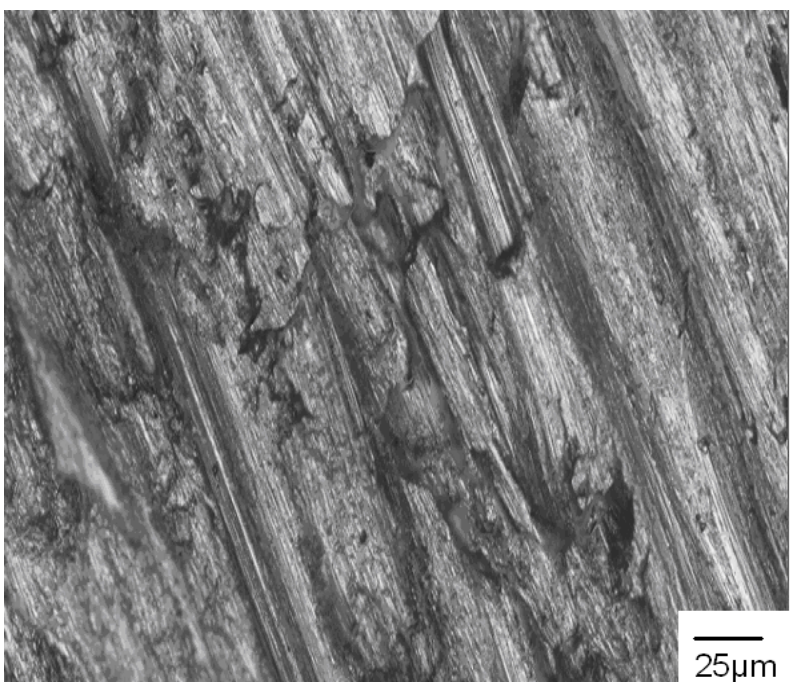

(a)

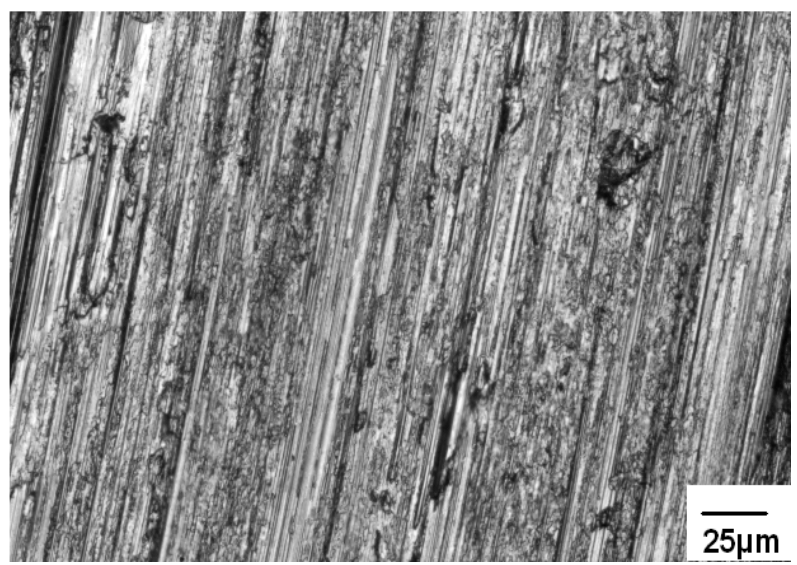

(b)

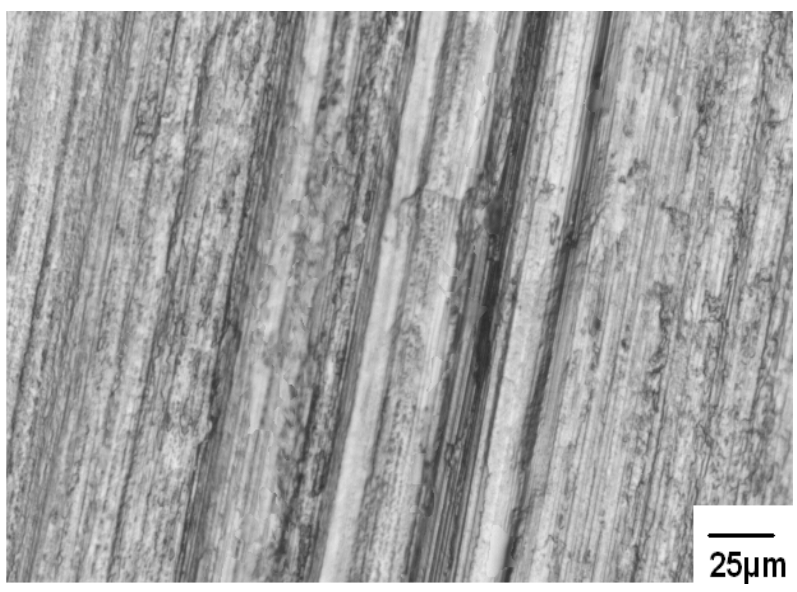

(c)

Figure 6. (a) SEM showing wear tracks in the unreinforced alloy; (b) SEM showing wear tracks in the $7 \%$ graphite reinforced non-nitrided composite; (c) SEM showing wear tracks in the 7\% graphite reinforced and nitrided composite. 
motes easy shear between the lamellar planes of graphite. The buildup of the film is a significant feature of graphite tribology. Graphite has a hexagonal layered structure and the bonds between the parallel layers are relatively weak (van der Walls type). The key to graphite's value as a self-lubricating solid lies in its layered lattice structure and its ability to form strong chemical bonds with gases such as water vapour. The adsorption of water vapour and other gases from the environment onto the crystalline edges weakens the interlayer bonding forces, resulting in easy shear and transfer of the crystalline platelets on to the mating surfaces. Graphite also performs well under boundary lubrication conditions because of its affinity fro hydrocarbon lubricants [10].

Hence it can be concluded that the ability of the sheared reinforcement layers to adhere to the sliding surface decides the effectiveness of the graphite particles in reducing the wear rate of the composite materials.

\subsection{Effect of Nitriding on Wear Rate}

Nitriding is a surface treatment process where nitrogen is supplied into the chamber of specialized equipment at relatively high temperature. A nitrided thin layer on the surface will be formed containing aluminum alloy based phases. The addition of graphite to the base alloy significantly improves the wear resistance. This is further improved by several folds in the case of nirtided composites. The hardness of the nitrided composites increases quite significantly as shown in the Table 2 . The primary cause for the increase in hardness and hence wear resistance is the presence of AlN and nitrogen enriched aluminum at the surface. The presence of the phases is shown by the XRD analysis which is presented in Figure 2(a).

\subsection{SEM Analysis}

In view of brevity and convenience, the SEM micrographs of only $7 \%$ composites at speed of $1.87 \mathrm{~m} / \mathrm{s}$ have been presented. However, the explanation holds good even for the composites with 3 and 5\% reinforcement as well. The SEM micrographs of a typical worn surface of the $7 \%$ graphite reinforced composites are presented in Figure 6(a), (b) and (c) which shows the wear track morphology of the specimens tested at various loads.

It can be seen that a lot of parallel, continuous and

Table 2. Vickers micro hardness number for nitrided and non-nitrided specimens.

\begin{tabular}{cc}
\hline Specimen Type & VHN \\
\hline Non-Nitrided Specimens & 68 \\
Nitrided Specimens & 82 \\
\hline
\end{tabular}

deeply ploughed grooves exist on the wear surface of the composites and there is an abrasion phenomenon observed at low loads. The parallel grooves suggest abrasive wear as characterized by the penetration of the graphite particles into a softer surface, which is an important contributor to the wear behavior of composites. The worn surfaces in some places reveal patches of material removed from the surface of the material during the course of wear and smeared on to the sliding surface.

\section{Conclusions}

The addition of graphite particles to the aluminum alloy improves the wear resistance of the composite in spite of the significant improvement to wear resistance. Nitriding which is a surface treatment process further improves the wear resistance by several folds. The primary cause for the increase in hardness and hence wear resistance is the presence of AlN and nitrogen enriched aluminum at the surface.

\section{REFERENCES}

[1] P. H. Chong, H. C. Man and T. M. Yue, "Microstructure and Wear Properties of Laser Surface Claded Mo-WC MMC on AA6061 Aluminium Alloy," Surface and Coatings Technology, Vol. 145, No. 1-3, 2001 pp. 51-59. doi:10.1016/S0257-8972(01)01286-5

[2] L. Liu, W. W. Li, Y. P. Tang, B. Shen and W. B. Hu, "Friction and Wear Properties of Short Carbon Fiber Reinforced Aluminium Matrix Composites,” Wear, Vol. 266, No. 7-8, 2009, pp.733-738. doi:10.1016/j.wear.2008.08.009

[3] Y. Sahin and K. Ozdin, "A Model for Abrasive Wear Behaviour of Aluminium Based Composites,” Materials and Design, Vol. 29, No. 3, 2008, pp. 728-733. doi:10.1016/j.matdes.2007.02.013

[4] A. T. Alpas and J. Zhang, "Effect of Microstructure and Counterface Materials on the Sliding Wear Resistance of Particulate Reinforced Aluminium Matrix Composites,” Metallurgical Transactions, Vol. 25, 1994, pp. 969-983.

[5] D. D. Nolan, S. W. Huang, V. Leskovsek and S. Braun, "Sliding Wear of Titanium Nitride Thin Film Deposited on Ti-6Al-4V alloy by PVD and Plasma Nitriding Processes," Surface and Coatings Technology, Vol. 200, No. 20-21, 2006, pp. 5698-5705. doi:10.1016/j.surfcoat.2005.08.110

[6] S. C. Sharma, B. M. Girish, D. R. Somashekar, B. M. Satish and R. Kamath, "Sliding Wear Behavior of Zircon Particles Reinforced ZA-27 Alloy Composite Materials,” Wear, Vol. 224, No. 1, 1999, pp. 89-94. doi:10.1016/S0043-1648(98)00334-2

[7] N. S. Poonawala, A. K. Chakrabarti and A. B. Chattopadhyay, "Wear characteristics of Nitrogenated Chromium Cast Irons,” Wear, Vol. 162, 1993, pp. 580-588. doi:10.1016/0043-1648(93)90544-V 
[8] P. K. Rohatgi, S. Ray and Y. Lin, "Tribological Properties of Metal Matrix Graphite Particle Composites,” International Materials Review, Vol. 37, 1992, pp. 129-138.

[9] P. P. Lee, T. Savaskan and E. Laufer, "Wear Resistance And Microstructure of $\mathrm{Zn}-\mathrm{Al}-\mathrm{Si}$ and $\mathrm{Zn}-\mathrm{Al}-\mathrm{Cu}$ alloys,"
Wear, Vol. 117, No. 1, 1987 pp. 79-84. doi:10.1016/0043-1648(87)90245-6

[10] Y. Sahin, “Tribological Behaviour of Metal Matrix and Its Composites,” Materials and Design, Vol. 28, No. 4, 2007, pp. 1348-1352. doi:10.1016/j.matdes.2006.01.032 